\title{
Chapter 8 Systemic Approach for the Definition of a Safer Human-Robot Interaction
}

\author{
Alessandro Pecora, Luca Maiolo, Antonio Minotti, Massimiliano Ruggeri, \\ Luca Dariz, Matteo Giussani, Niccolò Iannacci, Loris Roveda, \\ Nicola Pedrocchi and Federico Vicentini
}

\begin{abstract}
Smart factories must speed up their processes to face new manufacturing challenges and, at the same time, demonstrate an extremely high degree of flexibility to reduce production costs and time. This kind of issues can be addressed by the cooperation between humans and robots in a mixed human-robot working environment. Robots have the compelling advantage of spatial precision and repeatability as well as the capability of applying defined forces. Humans, on the other hand, are especially skilled at complex manipulations and adapting to changing task requirements. In this complicate scenario of co-shared workplace and continuous human-robot interaction, safety strategies are a key requirement to avoid possible injuries to humans or fatal accidents. This chapter proposes a systemic approach to respond to these requirements. The approach merges and manages multiple sensing sources, redundant transmission protocols and software decision mechanisms, aiming to guarantee a continuous and reconfigurable co-share scenario that enables an operative interaction between human workers and robots in a controlled and safe environment. Furthermore, new technological solutions and innovative methodologies are presented for the definition of a safer workplace in human-robot interaction scenarios.
\end{abstract}

\subsection{Scientific and Industrial Motivations}

The need to face the production of complex families of product, with short life cycles, often characterized by strongly variable production patterns, requires factories to be able to quickly evolve and reconfigure [1]. These reconfigurable factories

\footnotetext{
A. Pecora $(\bowtie) \cdot$ L. Maiolo $\cdot$ A. Minotti

CNR-IMM, Istituto per la Microelettronica e Microsistemi, Rome, Italy

e-mail: alessandro.pecora@cnr.it

M. Ruggeri · L. Dariz

CNR-IMAMOTER, Istituto per le Macchine Agricole e Movimento Terra, Cassana, FE, Italy

M. Giussani $\cdot$ N. Iannacci $\cdot$ L. Roveda $\cdot$ N. Pedrocchi $\cdot$ F. Vicentini

CNR-STIIMA, Istituto di Sistemi e Tecnologie Industriali Intelligenti per il Manifatturiero

Avanzato, Milan, Italy

(C) The Author(s) 2019

T. Tolio et al. (eds.), Factories of the Future,

https://doi.org/10.1007/978-3-319-94358-9_8
} 
must exhibit strong modularity and adaptability to exogenous and endogenous events $[2,3]$. In accordance with production dynamics, the design of workplaces within factories must take into account the current social and demographic trends (e.g. increase in average life expectancy and increase in retirement age). Attention to issues such as safety, education and ergonomics can also be the key lever to improve productivity and profitability of the factory.

Traditionally, factory design has clearly distinguished between the roles and areas of expertise of human operators and automated and/or robotized production cells. Unfortunately, this operative model is nowadays obsolete since it cannot react and quickly adapt to the incoming opportunities arising from the global market. In this new scenario, it is crucial to provide innovative paradigms that ensure an efficient and safe work environment, as well as comfortable and stimulating for human workers. The new factories that make cooperation between humans and automated devices will be able to cope with difficult production scenarios characterized by products with short life cycles and high variability, thus requiring a fast adaptation of production capacity and the development of new knowledge [4].

The research presented in this chapter will specifically address the topic of designing and implementing human worker and robot cooperating systems. In particular, design methodologies, technologies and devices will be explored to guarantee a safe and fruitful human-robot interaction. To this aim, it is necessary to address themes related to the study of man-machine interactions, and hence the modelling of work areas and their perception by robots using advanced sensing systems, thus creating safe hybrid work environments. Indeed, the achievement of a strong interaction between a robot and a human worker, in a completely safe environment, relies on the development of a suitable framework including sensing technology, system architecture and proper cognitive models [5]. A sensor network must provide a sufficient amount of information to the robot about the surroundings and these data must be reliably transmitted and interpreted by the machine to adopt the right behaviours. Moreover, the suitable sensing technology must be shared and it has to be portable, light and easy to wear so that the worker can comfortably operate in the workplace [6].

As case study, the innovative approach will be proposed in a fenceless assembly/disassembly scenario considering peculiar issues in different workcells and along different workflows processes. Especially in disassembly processes, in fact, many diverse tasks must be executed according to local workflow, adapting the control settings for general and local motion/interaction planning with a high grade of versatility. Such a complex ensemble of abilities from both sides (robots and human workers) requires a transparent and seamless dynamic modification of the interaction at a system level in which human workers can suitably exchange tasks, enter/exit parts of the task execution, find a due - i.e. safe and comfortable-behaviour of the robots, thus avoiding any possibility of injuries or uneven unexpected robot behaviours. This contextual analysis represents a significant working environment since it joins a high level of adaptability and variability of processes, parts, manufacturing conditions, specific mechanical parameters and, more critically, unique human-robot association modes and assignments in assisted/cooperative mechanised manufacture [7]. 
In the next sections, the development of a full strategy to improve safety in typical fenceless scenarios will be described and examined starting from the concepts of dynamic supervision and adaptation of the interaction and combining multiple sensing sources with a novel WPAN (Wireless Personal Area Network) extended in the robot area. The proposed innovative approach will face also the development of algorithms and cognitive systems for the perception and representation of spacetime environment of the factory and for the implementation of automatic processes of decision and action.

\subsection{State of the Art}

The first layer to build a systemic approach for the definition of a safer human machine environment relies on the possibility to maximize and diversify the different sensor sources deployed both on the robot and on the worker with the intent of providing a continuous feedback related to the position of the worker and his tasks. However, the presence of various materials (conductive, insulating, magnetic, etc.) in a specific workplace and the consequent issue related to anomalous absorbance in the electromagnetic spectrum still represents a critical aspect in the creation of a reliable set of data for the robot. In the years, many different sensing solutions have been proposed to overcome these issues and most of them rely on the use of multiples cameras and microphones [8]. Unfortunately, these technologies are limited by the line of sight of the associated camera or produce signals that are difficult to interpret for the robot. A further possible solution considers the specific operation distance between the worker and the robot (short and long-range). In case of long-range sensing technology, radio-frequency identification technology (RFID) has recently received a great attention due to manufacturing costs, power consumption, sensors wearability, response time, etc. [9]. Indeed, the tags embedded in worker smart garments (gloves, suite, etc.) can provide a reliable information of a tracked movement, without hindering the worker operation. Robots can use the signals coming from the RFID sensing technology to properly interpret the movements of the human worker and to consequently adapt their behaviour. However, also in RFID technology, different problems can arise from the signal detection at particular angle and from the specific distance between the tag and the reader involved in the localization process and the signal can be misinterpreted by the robot [10].

To properly manage and transmit the data produced by all the sensing networks, a robust wireless infrastructure needs to be adopted. In this case, the identification/recognition system identifies the operator presence and position in the robot area, using the combination of safe wireless channels and of unsafe low power wireless communication protocols (like the Ultra-low power Bluetooth end Zigbee). This process works both acquiring presence of communication link between the access points in the robot area and the units directly in the operator body, and applying an algorithm featuring the geo-referenced position of the operator based on the signal reception power from the operator unit. A deep analysis on communication physical 


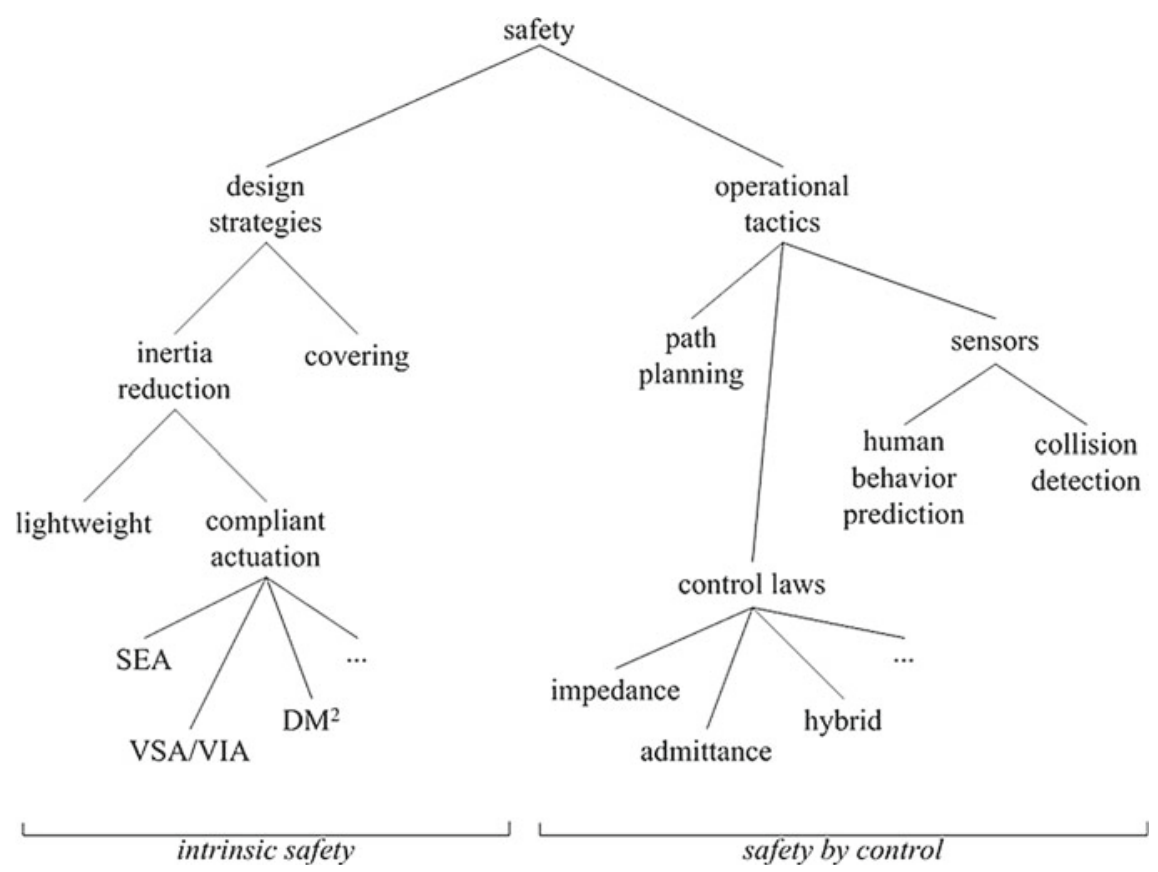

Fig. 8.1 Map of hardware features or design strategies that are used for embedding safety in robot systems

characteristics, power consumption, power management, easiness of reception power recognition systems, transceiver integration, availability and robustness in respect to industrial environment, must be taken into account to provide reliable data between the robot and the human workers.

Flexible/reconfigurable production, specifically when manual tasks can be assisted by robots, is remarkably supported by collaborative robotic methodologies that can guarantee a safe behaviour in unstructured dynamic environments. Lightweight solutions specifically designed for physical Human-Robot Interaction (pHRI) can be employed for these tasks. Since years, both academic [11-13] and manufacturing settlements $[14,15]$ have given careful consideration to broadening the coordinated effort space amongst robots and workers in industrial robotics as well. Industrial robots, from small to large size manipulators (e.g. anthropomorphic, Cartesian, parallel, etc.) still do not extensively display native safety features, either by design or by control (see Fig. 8.1). Nevertheless, such platforms are required to be integrated in safe setups at both machine and plant level.

On the normative side, most recent standards define human-robot cooperation modes and happen to apply on top of the consolidated standardization initiatives in machinery and robotics [16-21] that encapsulate the concept of functional safety altogether. Furthermore, safety is obviously an integrated concept, whose procedu- 
Fig. 8.2 Dependability chart

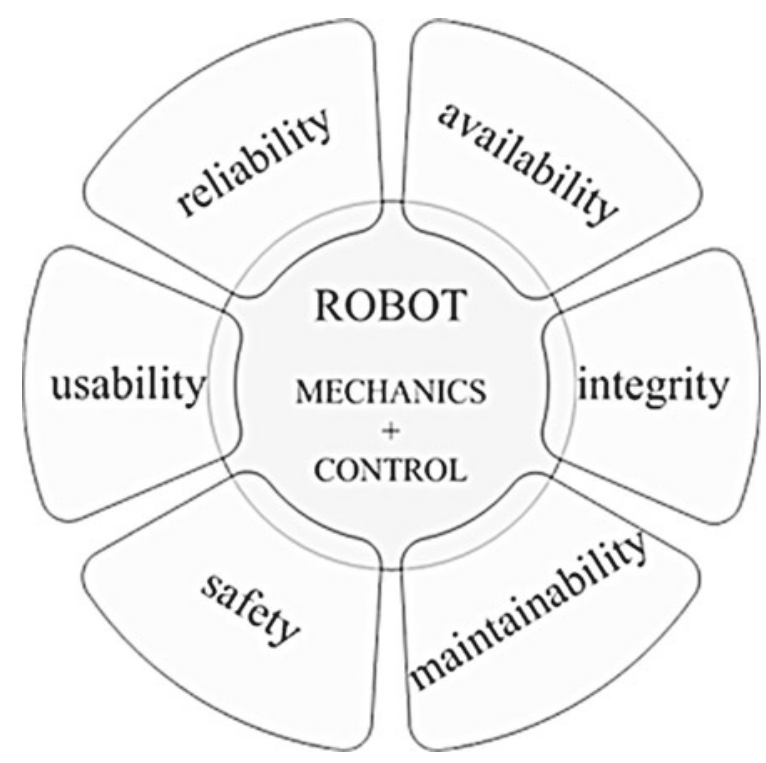

ral, logical and technological aspects are spread among hardware, algorithms and networks.

Safety options of devices are in all probability among the foremost self-addressed by technology suppliers due to a consolidated regulation $[16,18]$ and restricted needs in terms of integration may hinder the safety certification grade.

The notion of interaction in robotics can span different spatial conditions, from distant functional inter-action to close contact. Non-contact interaction is involved in the task cooperation and scenarios where the same workspace is concurrently visited. Contact interaction can occur either by planning or by accident. The former is primarily involved in most of the low-power tasks, like manipulation, handling, assembly, etc. where compliant controls represent a prominent class of settings. The latter has been considered as the major issue to be discussed and formalized in relationship with the normative requirements of safety, the very wide family of collision avoidance algorithms, etc.

At all interaction levels the cooperating robots should be transparent, i.e. they should resemble a naturally interacting companion, removing all cumbersome configurations or access modes, uneven motions or patterns, etc. This behaviour can be formulated through the concept of dependability that is considered as the property of displaying easiness, confidence and reliance to the user when performing interaction tasks. Under such conventionally abstract notion, dependability wraps a set of clearly defined child properties (see Fig. 8.2) [22] that altogether have a great impact over the robot control and resulting behaviour.

User confidence, as a matter of fact, stands on: 
- usability, which can measure the feeling, comfort and easiness of learning and/or setting up the robot motion,

- reliability, which mainly addresses the continuity of service rarely throwing unexpected exceptions or rarely presenting failures,

- availability, which gives account of readiness of such service,

- maintainability and integrity, which are the ability to recover from system alteration and the absence of improper alterations, respectively,

- safety, which has been extendedly discussed before.

Most of dependability child properties can be fulfilled as a trade-off at whole system level. The dependability in the interaction, and its effects on the dynamic allocation of robot behaviour, relies on the cognitive aspects of the human robot interaction. Ongoing studies in the field of brain research have concentrated on psychological procedures of joint-activity among people to empower a successful cooperation [23]. Psychological investigations demonstrate that an effective collaboration in human teams requires members to arrange and execute their activities while anticipating the actions of the other colleagues, and not simply reacting to them [24]. Therefore, a proficient human/robot collaboration needs an effective communication [25-27]. The benefit of anticipatory actions in a human/robotic environment is proven in [28], showing a significant improvement of task efficiency compared to a reactive behaviour. A shared representation of human and robot abilities must be available to properly assign the tasks to the partners in the working group [29, 30]. Human-Robot collaboration using peer-to-peer approaches is a key research topic in robotics [31]. Laengle et al. presents a human-robot team employing a multi-agent control architecture [32], where the mobile system consists of an overhead-camera and a two-arm-manipulator. A proactive collaboration based on the recognition of intentions is proposed in [33]. Intention is a state of mind of the human that cannot be measured directly. However, human action is a result of intention, so Dynamic Bayesian Networks (DBNs) can be used to cope with these uncertainties [33]. The approach in [29] presents a scenario where a human and a robot system with two robotic arms cooperate to build a wooden model of an aircraft using a cognitive architecture consisting of high-level components input, interpretation, representation, reasoning, and output with several functional modules.

The execution of interactive tasks in an assembly cooperative workcell relies on measurements from distributed sensors that return system and users information.

\subsection{Problem Statement and Proposed Approach}

The proposed robot control architecture aims at providing safe collaborative behaviours of the robots that are sharing the workspace, or physically interacting with human operators. The technological limits for enabling a multi-model collaboration (i.e. contact-less or physical interaction) with robots, are mainly deriving from the lack of real-time, failsafe tracking of humans inside shared workspaces. 
Additionally, all functions relevant to safety are required to comply with functional safety requirements.

The approach for solving the safe workspace sharing problem is then mainly based on the design of an architecture where all necessary sensing/control devices are integrated and synchronized, and that must be able to display failsafe properties.

The central infrastructure is a robotic Networked Control System (NCS, see Fig. 8.3) where tangible data are given by a heterogeneous arrangement of sources, including the sensors, diverse computational agents for the elaboration of functional application information and possible comparisons with stored data. The NCS provides an infrastructure for integrating general devices, including not safety-rated components. Fail-safe characteristics of the NCS architecture are based on several layers of cross-checking and packets validation data to decrease the probability to miss any detection of dangerous faults. Regarding functional safety, the target achievement is to reduce the Probability of Dangerous Failures per Hour $\left(\mathrm{PFH}_{\mathrm{d}}\right)$ to negligible levels, in particular corresponding to ISO 13849 PLd, which is in general the normative requirement for industrial robots. Elements in a NCS are not required to be secure in standalone mode, but their data must be monitored and validated online.

This approach permits to make transparent and continuous changes in the robot behaviour, as long as such algorithms are always protected from data or protocol failures. In particular, the most relevant components in the NCS will be presented in the next section and can be anticipated as follows:

- The functional design and development of the architecture of unsafe devices to synchronize and process data coming from the localization sensing arrays and from robots. An outline of the architecture design is provided in Sect. 8.4.1.

- Design and development of ad hoc WAN (Wide-Area-Network) network and WPAN (Wireless Personal Area Network) networks with an enhanced level of redundancy of data. This set of protocols enables the interconnection of remote sensors into the architecture. Details are provided in Sect. 8.4.2.

- The input for localizing human operators, to be integrated in the safety architecture, deriving from general purpose sensors. The design and integration of innovative wearable smart sensing systems for long range (less than $2 \mathrm{~m}$ ) and short range (less than $0.5 \mathrm{~m}$ ) localization to be worn by the human operator are described in Sect. 8.4.3.

In case of long range sensor technology, the requirements based on low power consumption and precision of human positioning addressed the choice to the usage of distance estimation through Received Signal Strength (RSS) measuring. This measurement represents the most accessible transmission parameter to estimate the distance between the nodes.

Wearable infrared devices, based on photoresistors with flexible thin film germanium sensing layer, will be presented for short range sensors [34, 35]. The system is conceived by housing an infrared source or multiple infrared sources of the robot and integrating the photoresistors directly on a work glove. The system automatically bal- 


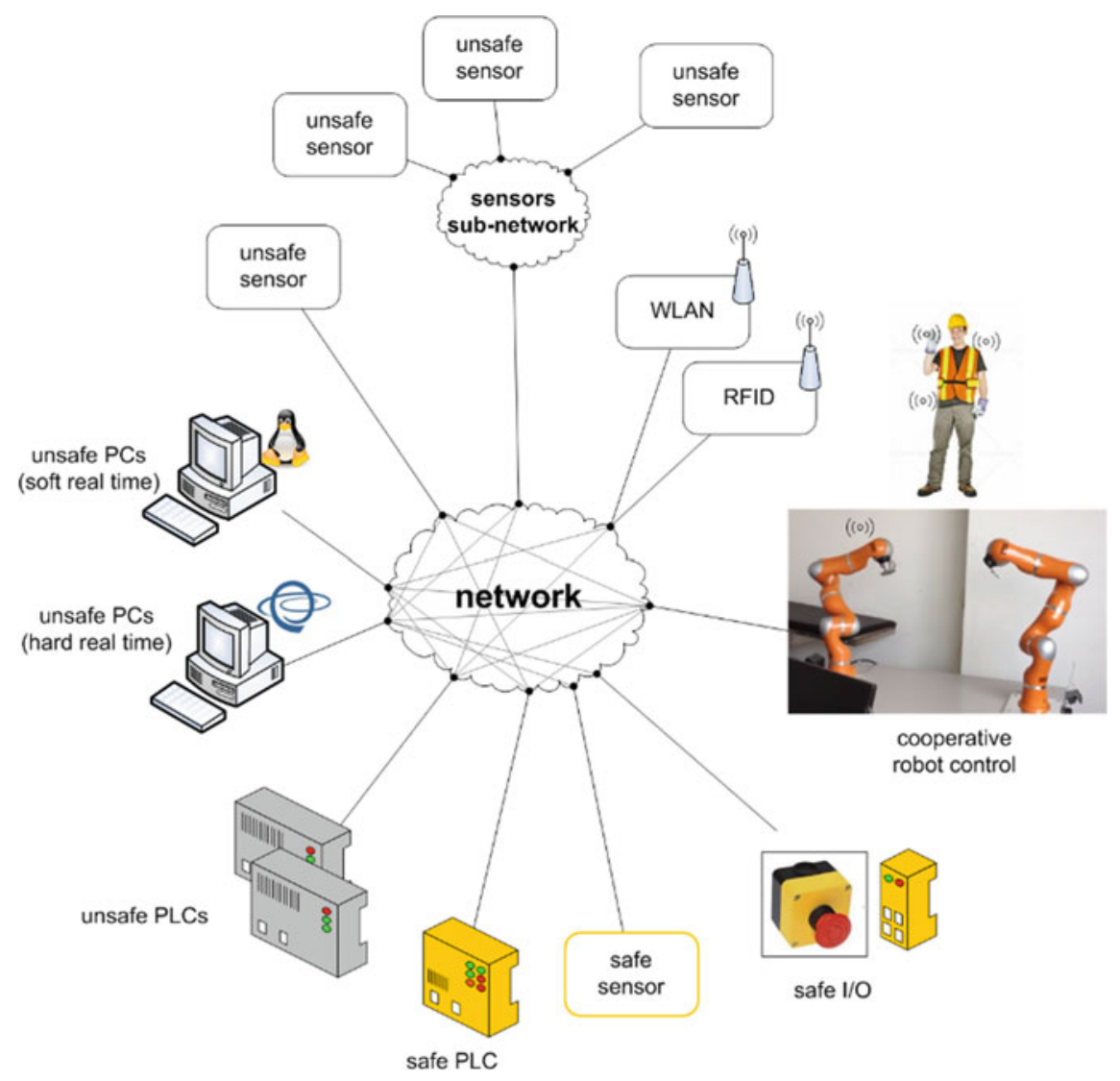

Fig. 8.3 Schema of a Robotic NCS. Safe-grade nodes (CPUs and sensors) are represented in yellow; CPUs can run different Operating Systems; sensors can be grouped in subnets

ances temperature variations and takes into account the infrared spatial distribution of emitting LED array.

\subsection{The Experimental Solution}

\subsubsection{Architecture for Safe Distributed Robotic Systems}

The core methodology for a safe distributed robot system consists in monitoring data channels and exchanged messages, to reduce the probability of failing in detecting data processed by sensor/monitoring nodes. Redundancy, diversity and monitoring are applied by a double independent elaboration of single channel sources. Then, the 

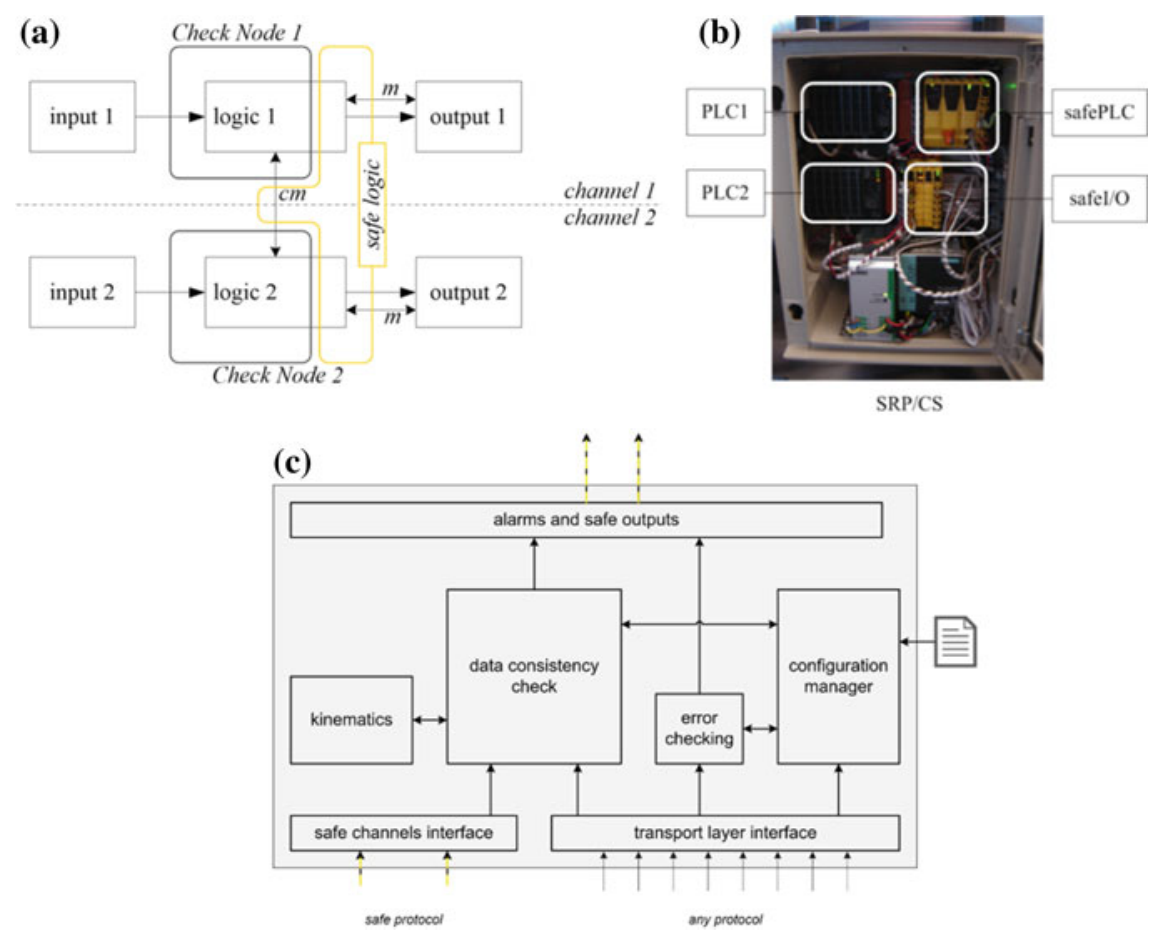

Fig. 8.4 a ISO13489-1:2006 model for Cat:3 architecture with deployed SRP/CS components (CPU1, CPU2 and safe CPU) outlining their logical domain. " $\mathrm{m}$ " are monitored safe state tasks execution. "cm" is the cross-monitoring of both channels. b Deployed SRP/CS featuring hardware components (PLC1, PLC2 and safePLC). c Architecture of the "logic" parts of the control system/safety related part (SRP/CS)

output data of comparative units (check nodes, programmed on standard PLC - see Fig. 8.4) are checked for consistency by a final safe node that plays the role of safety gate between the safety functions domain and all the general purpose CPUs or the unsafe sensors. Architecturally, the Safety-Related Part of a Control System (SRP/CS) is distributed in three logical components: two check-node CPUs, capable to support standard floating-point computation and to connect to user-defined libraries, and a component embedding a safe logic that is suitable to fulfil the preliminary conditions of dual structure and availability of monitoring coverage [36-38].

Components integrated in the NCS architecture can track devices and robots, using both wired and wireless communications infrastructures (see Fig. 8.5). In particular, the focus was on the verification of the communication interfaces (WLAN and UDP/IP) and analysis/validation of the specific parameters of the subsystems, including failures detection and protocol performance. 


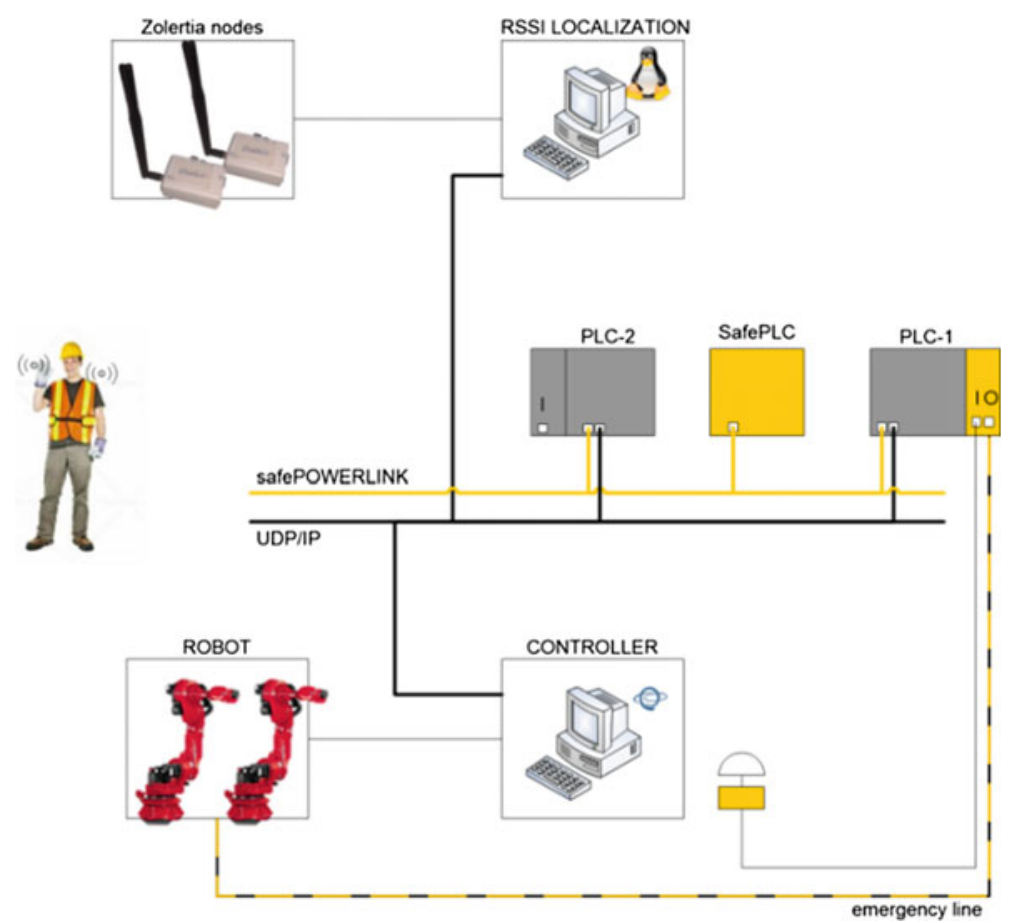

Fig. 8.5 Layout, devices and connections for the solution design [38]

\subsubsection{Gateway Architecture for Wireless Node in the NCS}

The Gateway Node is composed of a wireless node, based on the IEEE 802.15.4 standard, which handles the communication schedule of the real-time Wireless Sensor Network and forwards the data to an embedded PC through a USB link, as visible in Fig. 8.6. The embedded PC then forwards the data through a wired Ethernet network, encapsulating the data on UDP packets. The Gateway sends the raw data to a Localization Node, implementing the localization algorithm described in Sect. 8.4.3.3, which itself sends the estimated position to the Check Nodes, which provide the interface with the robot control network.

Two alternative scenarios have been considered as shown in Fig. 8.7.

With the first approach (Extended architecture in Fig. 8.7a), the Gateway Node must act as a bridge, i.e. it reads data from the real-time WSN, changes the safety header and forwards the packets to the Ethernet interface towards a separate Localization Node. Conversely, with the second approach (Reduced Architecture in Fig. 8.7b) the Gateway Node embeds the functionality of Localization and Check Node. 
8 Systemic Approach for the Definition of a Safer Human-Robot ...

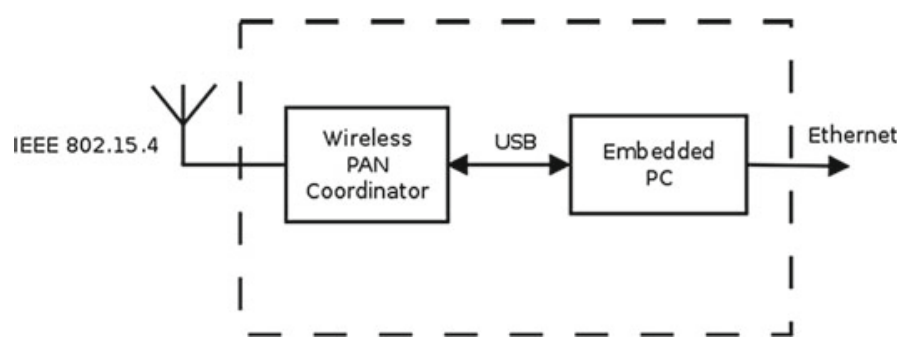

Fig. 8.6 Block diagram of the Gateway Node

(a)

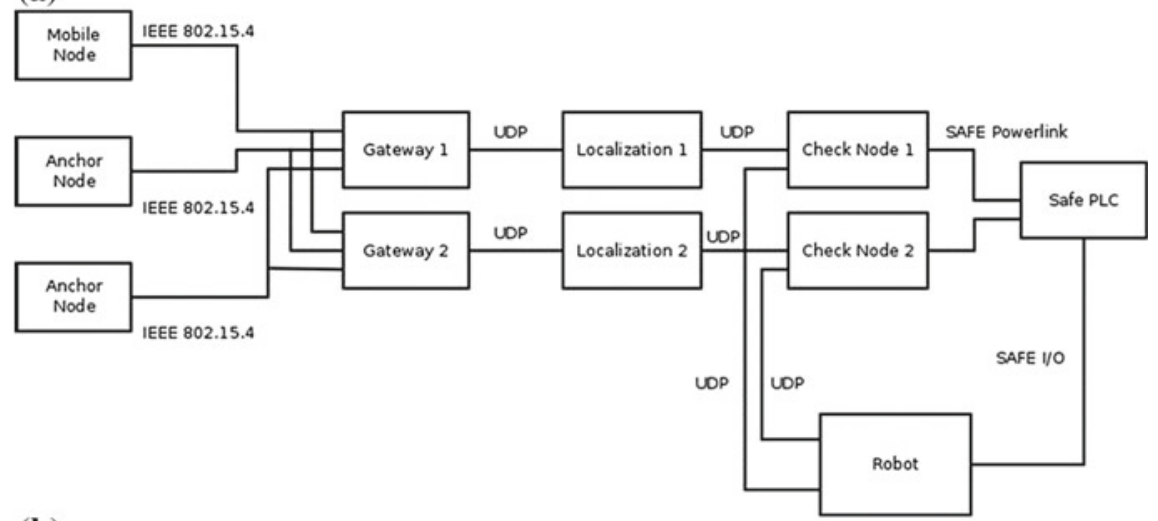

(b)

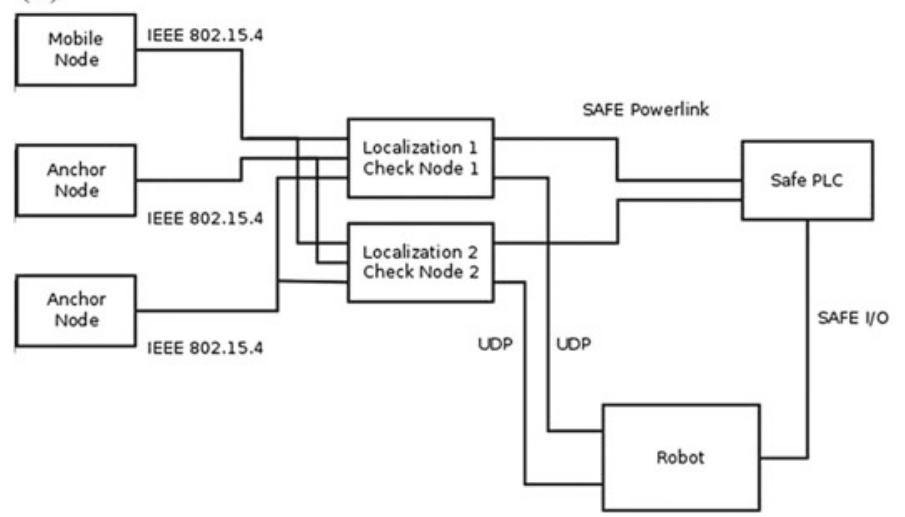

Fig. 8.7 a Extended architecture, b Reduced architecture 
The choice between the two architectures depends on the computational power available on the Gateway Node; in the integration experiments the Gateway node was realised first with a low-power and low-performance embedded board (Zolertia Z1 ${ }^{1}$ ), therefore corresponding to the Extended Architecture, and subsequently with a more powerful embedded board (Olimex P207²) that includes an Ethernet connection.

At the safety level the communication happens between the Wireless Sensor Nodes and the Localization Node through a safe communication channel. Data collected from the safe channel are used to compute the estimated position of the Mobile Node and to check errors that can occur, due to the intrinsic characteristics of the wireless channel. The localization algorithm should therefore not use expired or duplicated data, check data integrity and promptly forward to the Check Node an error status when it is not possible to reliably collect enough data from the Wireless Sensor network to perform the localization algorithm.

\subsubsection{Software Stack for Real-Time Wireless Sensor Network in the NCS}

The communication stack is based on IEEE 802.15.4e-LLDN (Low Latency Deterministic Network), with some modifications that enable to further reduce the worstcase communication latency. The code was initially designed to run on an embedded microcontroller without an operating system, subsequently it has been ported to a real-time operating system, which is needed to satisfy more strict real-time constraints. The software is composed of a high level Medium Access Control (MAC) and a low level MAC; the former controls the content of the packets, the configuration and the management of the network, while the latter handles the transmission and reception of the packets (see Fig. 8.8). The software is modular and can be easily ported to other platforms; as an example, it has been ported to an ARM Cortex-M3 and a discrete-time network simulator (NS3). The reference implementation is based on a commercial off-the-shelf wireless sensor network module, the Zolertia Z1, with a 16-bit RISC microcontroller (MSP430) running at $16 \mathrm{MHz}$.

The implementation presents some optimizations compared to the standard LLDN mode that reduce the maximum worst-case latency of the communication on the wireless channel and the time of system configuration; conversely the number of nodes that can be connected to the network with the same performance are also reduced [39-41].

The communication between the sensor nodes and the gateway node (Personal Area Network coordinator), according to IEEE 802.15.4e LLDN specifications, is synchronized through a temporal structure called superframe, which implements a Time Division Multiple Access method (TDMA).

\footnotetext{
${ }^{1}$ https://github.com/Zolertia/Resources/wiki/The-Z1-mote.

${ }^{2}$ https://www.olimex.com/Products/ARM/ST/STM32-P207/.
} 
8 Systemic Approach for the Definition of a Safer Human-Robot ...

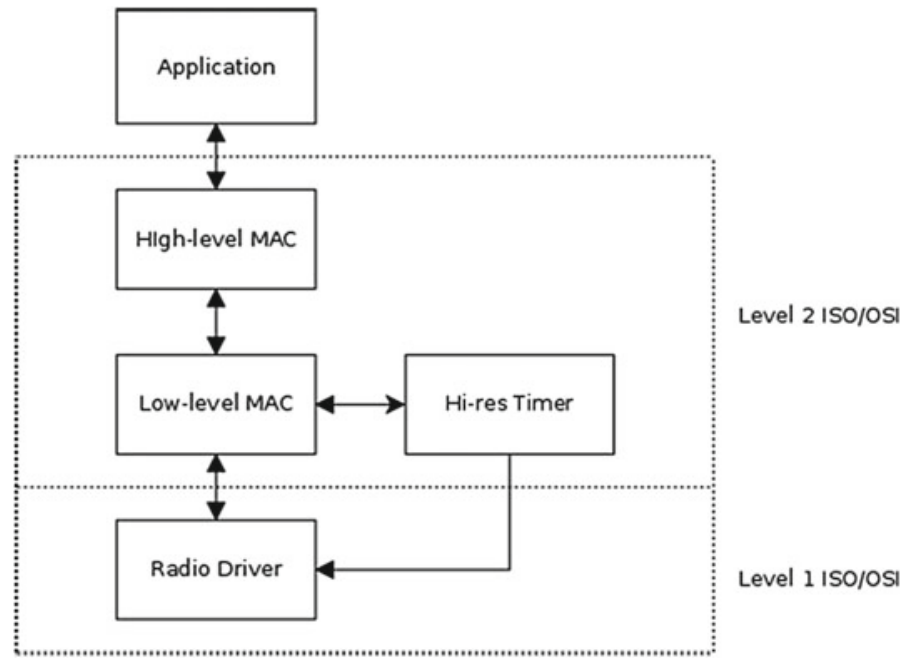

Fig. 8.8 Architecture of the wireless sensor network communication stack

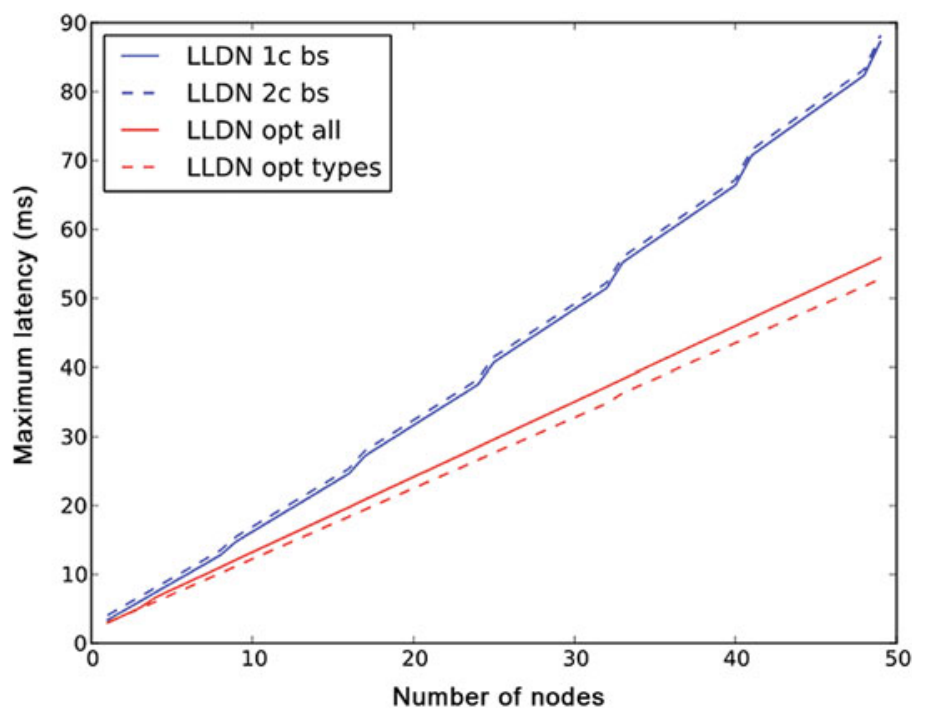

Fig. 8.9 Maximum latency achieved with sensor nodes and anchor nodes. It can be seen the net improvements in terms of time cycle in the regime phase of the network system thanks to the allocation of time slots 

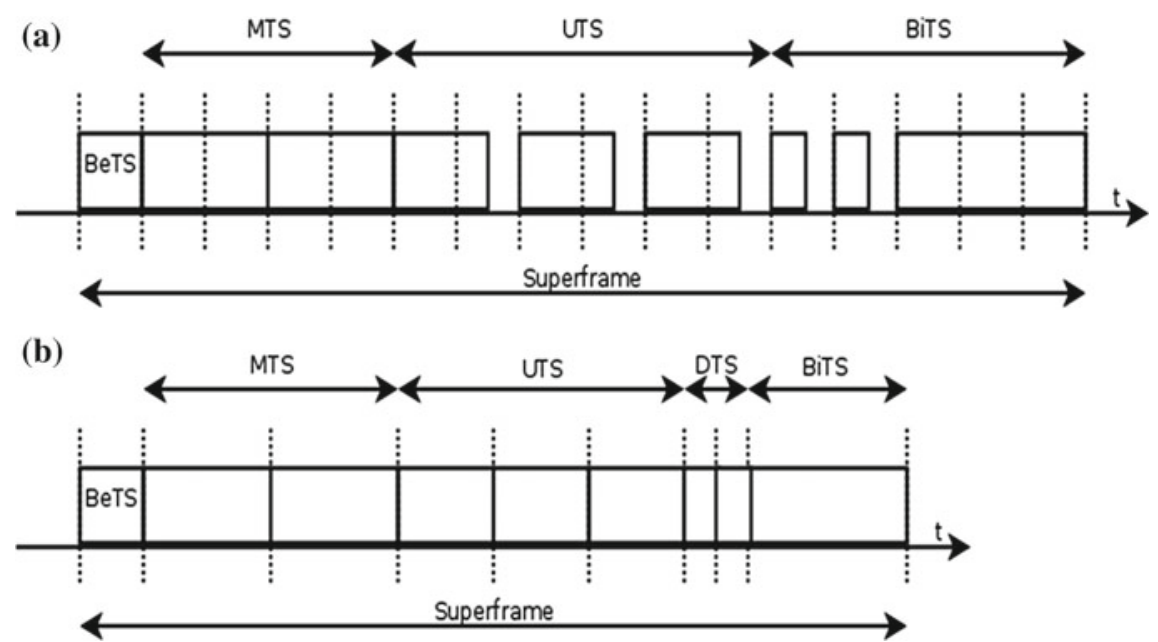

Fig. 8.10 a Standard IEEE 802.15.4 LLDN superframe format; b Proposed modified superframe format

Table 8.1 Superframe time for Standard and Modified LLDN for 10 and 20 nodes in a real-time WSN [38]

\begin{tabular}{l|l|l}
\hline \# Nodes & $\begin{array}{l}\text { Superframe time }[\mathrm{ms}] \\
\text { Standard LLDN }\end{array}$ & $\begin{array}{l}\text { Superframe time }[\mathrm{ms}] \\
\text { Modified LLDN }\end{array}$ \\
\hline 10 & 22.1 & 17.3 \\
\hline 20 & 41.3 & 32.7 \\
\hline
\end{tabular}

Figure 8.9 shows a comparison between two standard LLDN configurations (in blue) and two optimized configurations (in red), where a reduce communication latency is possible with the same number of nodes on the WSN. Figure 8.10 shows the comparison between a standard LLDN superframe structure and the superframe structure of the optimized LLDN protocol, which is the reason for the performance improvement showed in Fig. 8.9. Some numerical results are reported in Table 8.1.

The Configuration phase of the superframe structure has also been optimized, obtaining a significant reduction of configuration time of the 802.15.4-based wireless network. This has been achieved through an exclusive access of the nodes to the channel in the initial setup of the network, where in standard LLDN a shared access method is used. This required the modification of the packets used to discover all the nodes present on a WSN. The optimized configuration phase has been simulated using a Monte-Carlo approach for the standard LLDN, to model the shared access method, while for the optimized LLDN only a deterministic computation is required.

Figure 8.11 shows a comparison of the Configuration time between Standard and Modified LLDN Protocol. 


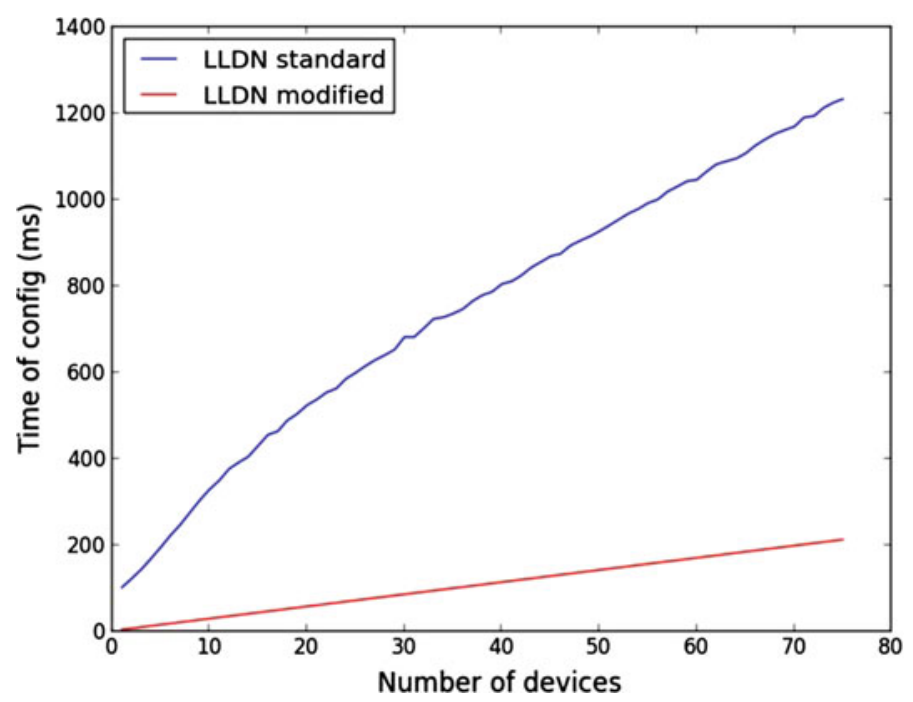

Fig. 8.11 Configuration Time for simulated Standard LLDN protocol, highlighting the time taken for procedure adhering to the standard (blue line) and calculated Modified LLDN (red line)

\subsubsection{Safety Layer for Wireless Sensor Network}

The safety layer implements the mechanisms needed to detect all the possible communication errors, thus enabling a reliable communication over a transmission channel that is intrinsically unreliable, such as the wireless channel. To check for transmission errors, a safety protocol has been designed on top of the layer 2 protocol used to access the wireless channel, by explicitly adding a timestamp to each message, a running number, an explicit acknowledgment and a time of validity, which combined together dramatically increase the reliability of the system. This enables the communication of safety-relevant data to and from a Wireless Sensor Network (WSN), according to the black channel principle. The counter measures adopted are represented in Fig. 8.12, showing how the possible transmission errors are detected. The safety layer is designed to minimize the added overhead, both in terms of packet length and complexity, so the resulting packet is well suited for the transmission over a Low-Rate WPAN with sufficient reliability; in particular, a compression of the timing information is necessary. The software prototype consists of a firmware part (source) and a PC part (sink), that are necessary to implement and monitor a reliable communication. 


\begin{tabular}{|c|c|c|c|c|c|c|c|}
\hline $\begin{array}{c}\text { Type of } \\
\text { error }\end{array}$ & running number & timestamp & $\begin{array}{c}\text { time } \\
\text { expiration }\end{array}$ & $\begin{array}{c}\text { reception } \\
\text { ack }\end{array}$ & $\begin{array}{c}\text { sender/receiver } \\
\text { id }\end{array}$ & $\begin{array}{c}\text { data integrity } \\
\text { assurance }\end{array}$ & $\begin{array}{c}\text { redundancy } \\
\text { with cross check }\end{array}$ \\
\hline unintegity assurance \\
systems \\
\hline loss
\end{tabular}

$x$ - The measure protects against the type of error

0. Implemented in the safety layer

Fig. 8.12 Sources of error messages on black channels and implemented countermeasures

\subsubsection{Sensing System}

As the input side of the safe infrastructure, a sensing layer has been adopted with the intent of providing in real time a continuous feedback between the worker and robot. In particular, the sensing layer is composed of two wearable prototypes: (1) a long-range detection system integrated into a safety jacket, (2) a short-range sensing platform mounted on work gloves.

In the first case, a set of five inertial modules provides the position of the work in real time and a central ZigBee unit guarantees the data transmission. All the electronics and wires are embedded in an internal pocket architecture, thus creating a soft housing for the system. Also the power supply, in form of a battery, is connected to the central ZigBee unit on the chest.

For the second prototype, standard work gloves have been modified in order to house flexible photoresistors based on germanium thin film technology. A sensor for each glove has been integrated together with the electronics to investigate the reliability of the system and the limits of this technology. The devices are conceived to interact with an infrared source placed in the central part of the robot, with the intent of monitoring a distance ranging from $30 \mathrm{~cm}$ to the contact.

\subsubsection{Smart Working Suit}

A specific working suit has been developed to capture the worker posture inside a production line. The suit has been endowed with several Magnetic Angular Rate and Gravity (MARG) sensors and a RF transmission module. The fusion of data coming from the MARG sensors enables to predict the relative position of the sensors 


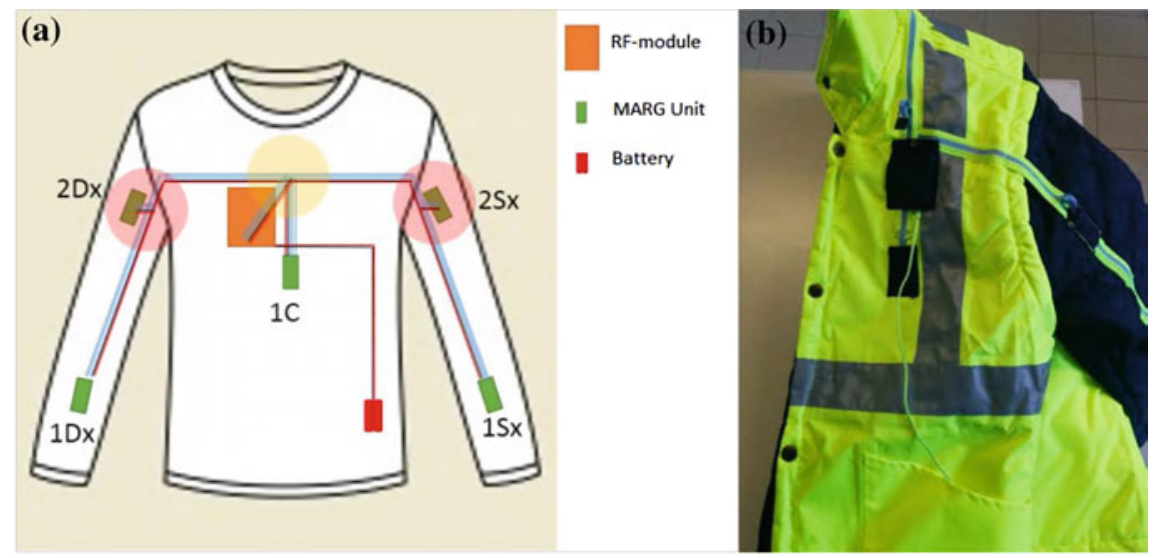

Fig. 8.13 a Schematic representation of the working suit. In the figure, MARG units, RF- module, battery and the wired connection are highlighted. b Photo of the assembled prototype

themselves and thus of the rigid body firmly bound to it, while the RF module is used both to transmit the MARG sensor data to the PC and as localization sensor. The MARG sensor units were placed on the arms, forearms and chest, to capture the motion of the human joints. The sensor units are connected by means of a shared serial bus directly integrated into the woven suit (see Fig. 8.13).

\subsubsection{Short Range Sensor}

A set of flexible infrared sensors has been fabricated to be housed on a glove to provide a valuable feedback for interactions between the worker and the robot in close proximity.

The prototype sensors have been developed using the RF-PECVD (Radio Frequency-Plasma Enhanced Chemical Vapour Deposition) technique. RF-PECVD enables to deposit materials on surface by means of vapour deposition of its gaseous state, in this case heating germane gas $\left(\mathrm{GH}_{4}\right)$ and blends of Germane-Silane $\left(\mathrm{SiH}_{4}\right)$ at $250-300{ }^{\circ} \mathrm{C}$. Changing the relative concentration of the two gases, it is possible to obtain devices with different characteristics, thus maximizing the sensing performance of the devices.

Finally, a simple read-out electronic has been designed and developed to perform a first stage of signal conditioning in the proximity of the sensor. In particular, an interface circuit for the resistive optical sensor in Germanium, has been designed and realized in through the use of technology based on thin film transistors (TFTs) of polycrystalline silicon at low temperature (LT-PS) deposited on the flexible substrate of polyimide (PI). 
(a)
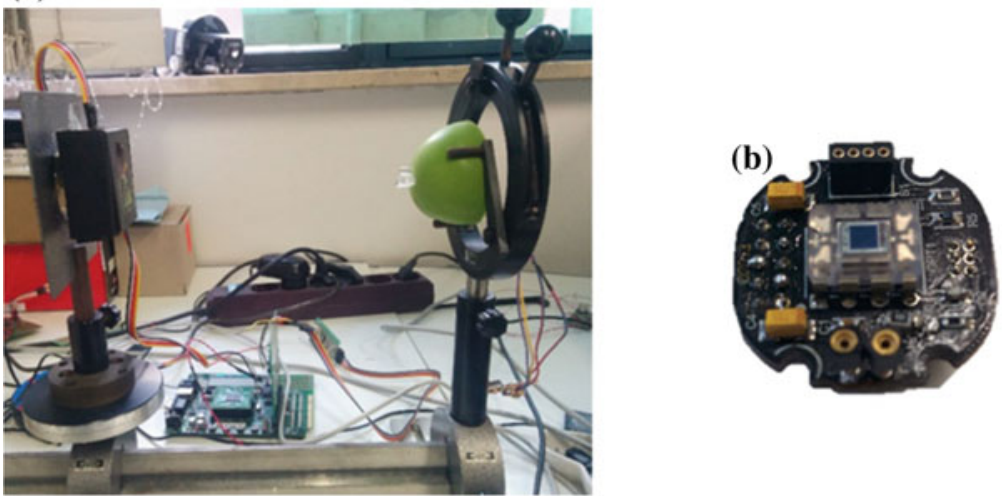

(c)

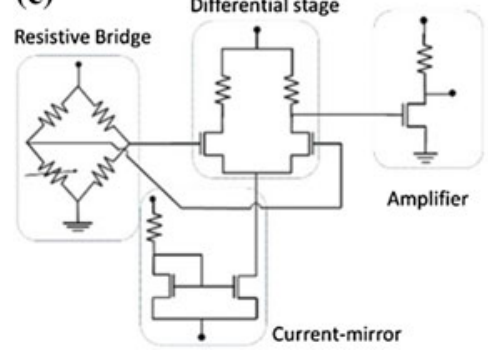

(d)

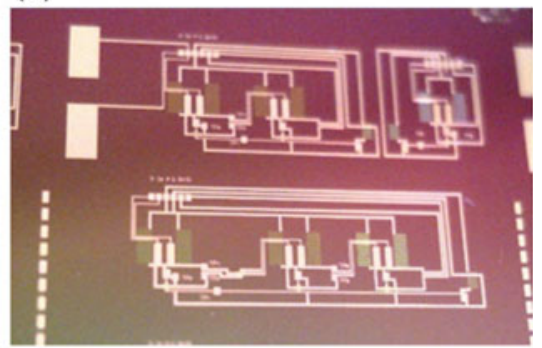

Fig. 8.14 a Graduated linear optical demonstrator mounted on a bench used to align first arrangement of IR sources and receivers. b Commercial IR-sensor. c Diagram of read-out electronic for the IR resistive sensors. d Photograph of the detail of the block of differential stages and current mirrors and Si-Ge IR-Sensor prototype

The circuit architecture is divided into several functional blocks: the first of these is a Wheatstone bridge to $1 / 4$, inside of which the optical sensor is inserted. The second functional block is constituted by the differential amplifier, fed by a current mirror. As the last step, a common-source amplifier allows to decouple the output of the differential stage from that of the circuit.

The entire circuit design was based on a process of n-MOS LTPS, (see Fig. 8.14c, d) [42]. To properly measure the sensors, preliminary tests have been carried out on a graduated linear optical bench. In the first step, a commercial IR sensor has been used to optimize the calibration system and then to realize a benchmark system for the prototype sensors (see Fig. 8.14a, b) [43, 44].

\subsubsection{Long Range Sensor}

According to the requirement of detecting operator position in a real industrial scenario with an average surface of about 25 square, a system equipped with protocol 

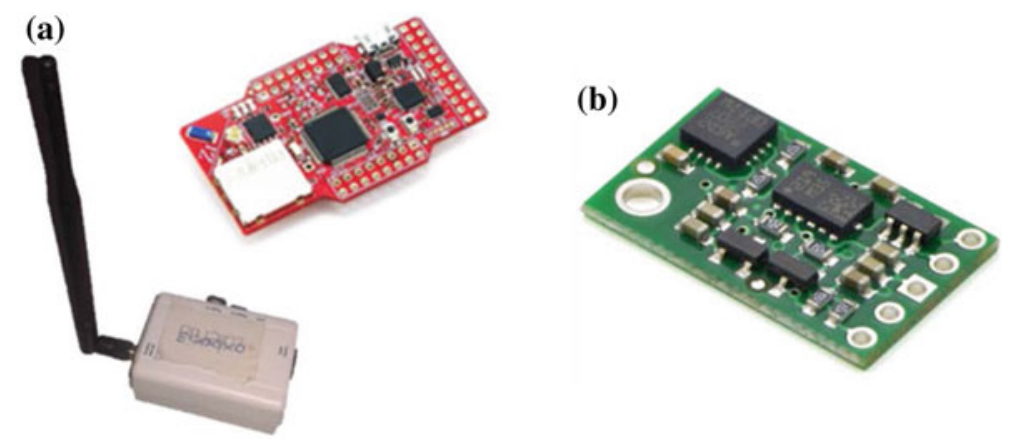

Fig. 8.15 a Zigbee Zolertia z1 node, b MARG Polulu inertial module (Size12.8 $\mathrm{mm} \times 20.3 \mathrm{~mm}$. Consumption $10 \mathrm{~mA}$ to $3.3 \mathrm{~V}$ )

ZigBee like has been adopted to evaluate in real time the interaction between the robot and the human worker. This system can exploit wireless localization by using different algorithms (typically Anchor based and Range based). In particular, the position detection platform consists of fixed reference nodes (anchor nodes), at known position, with which it is possible to evaluate the dynamic distance of the human worker by measuring the Received Signal Strength between the different radio frequency modules.

This localization technique is based on a triangulation method: in this case the detection of the distances between the object and at least three non-collinear reference anchors, permit to evaluate the relative position by using a suitable Lateration algorithm.

The developed localization algorithm consists of three phases: (1) ranging, in which distances are computed, (2) positioning, where nodes positions are estimated and (3) refinement, where the error is reduced through iterative methods. The solution chosen to implement the wireless sensor network for the localization, makes use of the commercial device Zolertia z1 (Fig. 8.15), that is a low-power module for sensor networks of small dimensions $(34.5 \times 56.8 \mathrm{~mm})$ and it is based on a TI MSP430 microcontroller, programmable in $\mathrm{C}$ language. The system is equipped with ZigBee module with integrated ceramic antenna. The card is completely expandable on account of the quantity of ports accessible (USB, I2C, SPI, 2xUARTs), so that inertial sensors and different other devices can be effectively externally connected.

The localization hardware system is very small in size and can be easily integrated in work clothes without being invasive. This system also lends itself to a more advanced integration in clothing, due to the relative simplicity of its electronic circuits, its open source type and completeness of the technical specifications that can be further miniaturized and made on thin, flexible PCBs.

Five inertial modules were connected to the mobile node to improve the assessment of the movement and position of the operator. These are placed on the upper limb (arm and forearm) and on the chest, for a total of five modules. The system is powered by $2 \mathrm{AA}$ batteries $3.3 \mathrm{~V}$ connected to the mobile node placed at the chest 
height. An inner pocket has been prepared to house the batteries and the connection wiring with the mobile node $[45,46]$.

The five inertial modules are connected to the mobile node with which they share the communication protocol and the power supply. The wires are five for each module, i.e. three wires (SDA and SCL I/O) and two power cords (3.3 V and GND).

The proposed system is able to recognize the position of a person in the space with an uncertainty of about $30 \mathrm{~cm}$. This value is perfectly compatible with the other recognition system based on short range sensors. This uncertainty strongly depends on the number of the fixed antennas located in the experimental space and it can be further reduced by adding heavier acquisition protocols. The position has been successfully acquired by real-time monitoring the movements of the person in a room equipped with different furniture and equipment made of metal and wood.

\subsubsection{Contact-Less Modes for Safe Workspace Sharing}

ISO/TS 15066:2016 defines the notion of Separation Monitoring that is intended to specify the concept of ISO 13855 minimum safety distance in the case of moving robots in the same workspace of human operators. Task-dependent safe distances are instead proportional to the current velocities of both robots and human operators. As a result, trajectory-dependent safety areas can be intuitively designed according to a given time-varying robot task, provided the availability of a safety-rated sensor system that measures relative positions and speeds. If the wireless sensors nodes (Sect. 8.4.3) are integrated in the overall architecture (Sect. 8.4.1) and use the proposed communication protocols (Sect. 8.4.2), then they support the dynamic computation of the minimum safety distance to maintain for the protection of the operator.

Safe emergency states or the violation of separation conditions can be triggered far less frequently than regular safeguarded cases, because the separation area is optimized (i.e. reduced) along the current task movement direction. The concrete result is that the entire virtual envelope of the protected space around the interacting bodies allows some proximity conditions that are useful for adaptability and efficiency in production.

Figure 8.16 shows that the human operator $\mathrm{H}$, while moving at a given velocity, is safe as long as it is not inside the red region that envelopes all the safe distances that separate $\mathrm{H}$ from all the parts of the robot $\mathrm{R}$. The shape of the safety region changes over time depending on the mutual H-R configurations. 


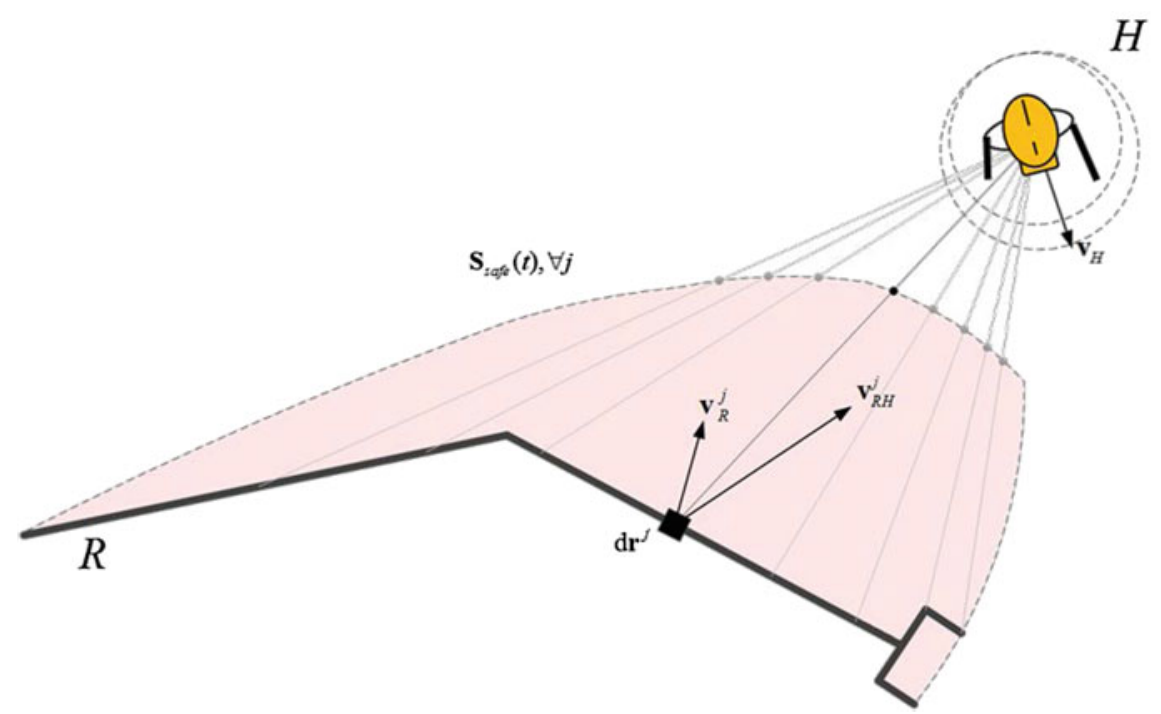

Fig. 8.16 Visualization of dynamic safe minimum distance. The red region minimizes the portion of the collaborative workspace that cannot be occupied by the user at a given time

\subsection{Conclusions and Future Research}

A multiple sensor network based on wearable and conformable technology represents a unique tool in advanced Wireless Sensor Network environment. Specifically, the implementation of WSN to track workers in joint human/robot workspaces has been presented as a source of environmental information that is merged with additional technologies to estimate risky conditions in collaborative tasks. With the aim to provide a logical settlement for safety-related operations, architectural solutions have been presented to deploy information, from unsafe sources to safe Inputs/Outputs. Proper network protocols, for easy and low-cost integration of general purpose sensors, are employed as black channels to connect safe processing units that are in charge of providing the required protection level. It has been shows that the modified WSN protocol can increase significantly the channel real time for upgrading and/or preserving the overall Safety Integrity Level. The enhanced protocol bridges some gaps in the service availability of 802.15 .4 protocol, thus fulfilling relevant solutions for safe applications in industry. While a full risk assessment procedure has to consider the analysis of faults and their severity, functional safety of communication protocols are considered beneficial because of the implementation of monitored failure modes, instead of unrecoverable dangerous failure modes, in standard communication networks [38].

These results will boost the interest of small industries and stakeholders for applications and technologies to be deployed in different scenarios from automotive assembly line to fenceless interactions between humans and robots in general. 
Even in different applications especially in biomedical market, wearable technology and prototypes could exploit the solutions proposed in this work to overcome several issue related to device durability, comfort, and contact force detection [47].

Acknowledgements This work has been funded by the Italian Ministry of Education, Universities and Research (MIUR) under the Flagship Project "Factories of the Future-Italy" (Progetto Bandiera "La Fabbrica del Futuro") [48], Sottoprogetto 1, research projects "FACTOry Technologies for HUMans Safety" (FACTOTHUMS) and "Improving human-robot cooperation and safety in shared automated workplaces of the automotive industry". The latter project was selected by the Canada-Italy Concurrent Call on Automotive Manufacturing R\&D and its twin Canadian project was funded by Automotive Partnership Canada to assess the usability of the technological and architectural solutions in a pre-industrial automotive scenario [43, 44].

\section{References}

1. Burke R, Mussomeli A, Laaper S, Hartigan M, Sniderman B (2017) The smart factory-responsive, adaptive, connected manufacturing. Deloitte University Press

2. Tolio T, Ceglarek D, Elmaraghy HA, Fischer A, Hu SJ, Laperriere L, Newman ST, Vancza J (2010) SPECIES-co-evolution of products, processes and production systems. CIRP Ann Manuf Technol 59(2):672-693

3. Terkaj W, Tolio T, Valente A (2009) Designing manufacturing flexibility in dynamic production contexts. In: Tolio T (ed) Design of flexible production systems. Springer, pp 1-18

4. Müller R, Vette M, Scholer M (2016) Robot Workmate: a trustworthy coworker for the continuous automotive assembly line and its implementation. Procedia CIRP 44:263-268

5. Burghart C, Mikut R, Stiefelhagen R, Asfour T, Holzapfel H, Steinhaus P, Dillmann R (2005) A cognitive architecture for a humanoid robot: a first approach. In: Proceedings of 5th IEEERAS international conference in humanoid robots, Tsukuba, Japan

6. Robla-Gómez S, Becerra VM, Llata JR, González-Sarabia E, Torre-Ferrero C, Pérez-Oria J (2017) Working together: a review on safe human-robot collaboration in industrial environments. IEEE Access 5:26754-26773

7. Tolio T, Copani G, Terkaj W (2019) Key research priorities for factories of the future-part I: missions. In: Tolio T, Copani G, Terkaj W (eds) Factories of the future. Springer

8. Athalye A, Savić V, Bolić M, Djurić PM (2011) A radio frequency identification system for accurate indoor localization. In: Proceedings of IEEE international conference on acoustics, speech and signal processing (ICASSP), Prague, Czech Republic

9. Bouet M, dos Santos AL (2008) RFID tags: positioning principles and localization techniques. In: Proceedings of IFIP wireless days 2008, Dubai, United Arab Emirates

10. Hagelauer A, Ussmueller T, Weigel R (2012) SAW and CMOS RFID transponder-based wireless systems and their applications. In: Proceedings of frequency control symposium (FCS), 2012 IEEE international, Baltimore, MD, USA

11. De Santis A, Siciliano B, De Luca A, Bicchi A (2008) An atlas of physical human-robot interaction. Mech Mach Theory 43(3):253-270

12. Pires $\mathbf{J}$ (2009) New challenges for industrial robotic cell programming. Ind Robot Int J 36(1)

13. Albu-Schaeffer A, Bicchi A, Boccadamo G, Chatila R, De Luca A, De Santis A, Giralt G, Hirzinger G, Lippiello V, Schiavi R, Siciliano B, Tonietti G, Villani L (2005) Physical human-robot interaction in anthropic domains: safety and dependability. In: Proceedings of 4th IARP/IEEE-Euron workshop on technical challenges for dependable robots in human environments

14. Behnisch K (2008) White paper-safe collaboration with ABB robots—electronic position switch and SafeMove. WHP-EPS-2006 
15. Matthias B, Kock S, Jerregard H, Källman M, Lundberg I (2011) Safety of collaborative industrial robots: certification possibilities for a collaborative assembly robot concept. In: Proceedings of IEEE international symposium on assembly and manufacturing (ISAM), Tampere, Finland

16. EN-ISO10218-1 (2011) Robots for industrial environments-safety requirements-part 1, Geneva

17. EN-ISO2018-2 (2011) Robots and robotic devices-safety requirements for industrial robotspart 2, Geneva

18. EN-ISO12100 (2010) Safety of machinery-general principles for design-risk assessment and risk reduction. ISO, Geneva

19. EN-ISO14121 (2007) Safety of machinery-risk assessment. ISO, Geneva

20. EN-ISO13849 (2006) Safety of machinery-safety-related parts of control systems. ISO, Geneva

21. EN-IEC61508 (2006) Functional safety of electrical/electronic/programmable electronic safety-related systems. ISO, Geneva

22. Vicentini F, Pedrocchi N, Molinari Tosatti L (2014) SafeNet: a methodology for integrating general-purpose unsafe devices in safe-robot rehabilitation systems. Comput Methods Programs Biomed 116(2):156-168

23. Lenz C, Nair S, Rickert AKM (2008) Joint-action for humans and industrial robots for assembly tasks. In: Proceedings of the 17th IEEE international symposium on robot and human interactive communication, Munich, Germany

24. Knoblich G, Jordan JS (2003) Action coordination in groups and individuals: learning anticipatory control. J Exp Psychol Learn Mem Cogn 29(5):1006-1016

25. Laengle T, Hoeniger T, Zhu L (1997) Cooperation in human-robot teams. In: Proceedings of the IEEE international symposium on industrial electronics, Guimaraes, Portugal

26. Breazeal C, Brooks A, Gray J, Hoffman G, Kidd C, Lee H, Lieberman J, Lockerd A, Chilongo D (2004) Tutelage and collaboration for humanoid robots. Int J Humanoid Rob 1(2):315-348

27. Sidner CL, Dzikovska M (2005) A first experiment in engagement for human-robot interaction in hosting activities. In: Advances in natural multimodal dialogue systems. Springer

28. Hoffman G, Breazeal C (2007) Effects of anticipatory action on human-robot teamwork: efficiency, fluency, and perception of team. In: 2nd ACM/IEEE international conference on humanrobot interaction (HRI), Arlington, VA, USA

29. Rickert M, Foster M, Giuliani M, By T, Panin G, Knoll A (2007) Integrating language, vision and action for human robot dialog systems. In: Proceedings of international conference on human-computer interaction, Beijing, PRC

30. Foster M, By T, Rickert M, Knoll A (2006) Human-robot dialogue for joint construction tasks. In: Proceedings of the international conference on multimodal interfaces. ACM Press

31. Tang F, Parker LE (2006) Peer-to-peer human-robot teaming through reconfigurable schemas. In: AAAI spring symposium on "To boldly go where no human-robot team has gone before", Stanford University

32. Längle T, Lueth T, Rembold U, Wörn H (1997) A distributed control architecture for autonomous mobile robots-implementation of the Karlsruhe multi-agent robot architecture (KAMARA). Adv Robot 12(4):411-431

33. Schrempf O, Hanebeck U, Schmid A, Worn H (2005) A novel approach to proactive humanrobot cooperation. In: Proceedings of IEEE international workshop on robot and human interactive communication, 2005, ROMAN 2005, pp 555-560

34. Chen E, Shih CY (2011) Polymer infrared proximity sensor array. IEEE Trans Electron Devices 58:1215-1220

35. Pecora A, Maiolo L, Maita F, Minotti A (2012) Flexible PVDF-TrFE pyroelectric sensor driven by polysilicon thin film transistor fabricated on ultra-thin polyimide substrate. Sens Actuators A Phys 185:39-43

36. Vicentini F, Pedrocchi N, Giussani M, Molinari Tosatti L (2014) Dynamic safety in collaborative robot workspaces through a network of devices fulfilling functional safety requirements. In: Proceedings of ISR/Robotik 2014; 41 st international symposium on robotics, Munich, Germany 
37. Vicentini F, Pedrocchi N, Molinari Tosatti L (2013) SafeNet of unsafe devices—extending the robot safety in collaborative workspaces. In: Proceedings of the 10th international conference on informatics in control, automation and robotics

38. Vicentini F, Ruggeri M, Dariz L, Pecora A, Maiolo L, Polese D, Pazzini L (2014) Wireless sensor networks and safe protocols for user tracking in human-robot cooperative workspaces. In: Proceedings of IEEE 23rd international symposium on industrial electronics (ISIE), Istanbul, Turkey

39. Dariz L, Ruggeri M, Malaguti G (2013) A proposal for enhancement towards bidirectional quasi-deterministic communications using IEEE 802.15.4. In: Proceedings of telecommunications forum (TELFOR), Belgrade, Serbia

40. Dariz L, Malaguti G, Ruggeri M (2014) Performance analysis of IEEE 802.15.4 real-time enhancement. In: Proceedings of IEEE 23rd international symposium on industrial electronics (ISIE), Istanbul, Turkey

41. Dariz L, Ruggeri M, Ferraresi C(2015) A comparison between configuration strategies for IEEE 802.15.4 low-latency networks. In: Proceedings of IEEE international conference on industrial technology (ICIT), Sevilla, Spain

42. Pecora A, Maiolo L, Cuscunà M, Simeone D, Fortunato G (2008) Low-temperature polysilicon thin film transistors on polyimide substrates for electronics on plastic. Solid State Electron $52: 348-352$

43. Ferrone A, Maiolo L, Minotti A, Pecora A, Iacovo D, Colace L, Grayli S, Leach G, Bahreyni B (2016) Flexible near infrared photoresistors based on recrystallized amorphous germanium thin films. In: Proceedings of 2016 IEEE SENSORS, Orlando, Florida, USA

44. Grayli S, Ferrone A, Maiolo L, Iacovo AD, Pecora A, Colace L, Leach G, Bahreyni B (2017) Infrared photo-resistors based on recrystallized amorphous germanium films on flexible substrates. Sens Actuators A Phys 263:341-348

45. Polese D, Pazzini L, Minotti A, Maiolo L, Pecora A (2014) Compensation of the antenna polarization misalignment in the RSSI Estimation. In: Proceedings of SENSORNETS 2014, Lisbon, Portugal

46. Polese D, Pazzini L, Minotti A, Maiolo L, Pecora A (2015) Tunable transmission power to improve 2D RSSI based localization algorithm. In: Proceedings of SENSORNETS 2015, 4th international conference on sensor networks

47. Ferrone A, Maita F, Maiolo L, Arquilla M, Castiello A, Pecora A, Jiang X, Menon C, Ferrone A, Colace L (2016) Wearable band for hand gesture recognition based on strain sensors. In: IEEE international conference on biomedical robotics and biomechatronics (BioRob), Singapore

48. Terkaj W, Tolio T (2019) The Italian flagship project: factories of the future. In: Tolio T, Copani G, Terkaj W (eds) Factories of the future. Springer

Open Access This book is licensed under the terms of the Creative Commons Attribution 4.0 International License (http://creativecommons.org/licenses/by/4.0/), which permits use, sharing, adaptation, distribution and reproduction in any medium or format, as long as you give appropriate credit to the original author(s) and the source, provide a link to the Creative Commons licence and indicate if changes were made.

The images or other third party material in this book are included in the book's Creative Commons licence, unless indicated otherwise in a credit line to the material. If material is not included in the book's Creative Commons licence and your intended use is not permitted by statutory regulation or exceeds the permitted use, you will need to obtain permission directly from the copyright holder. 